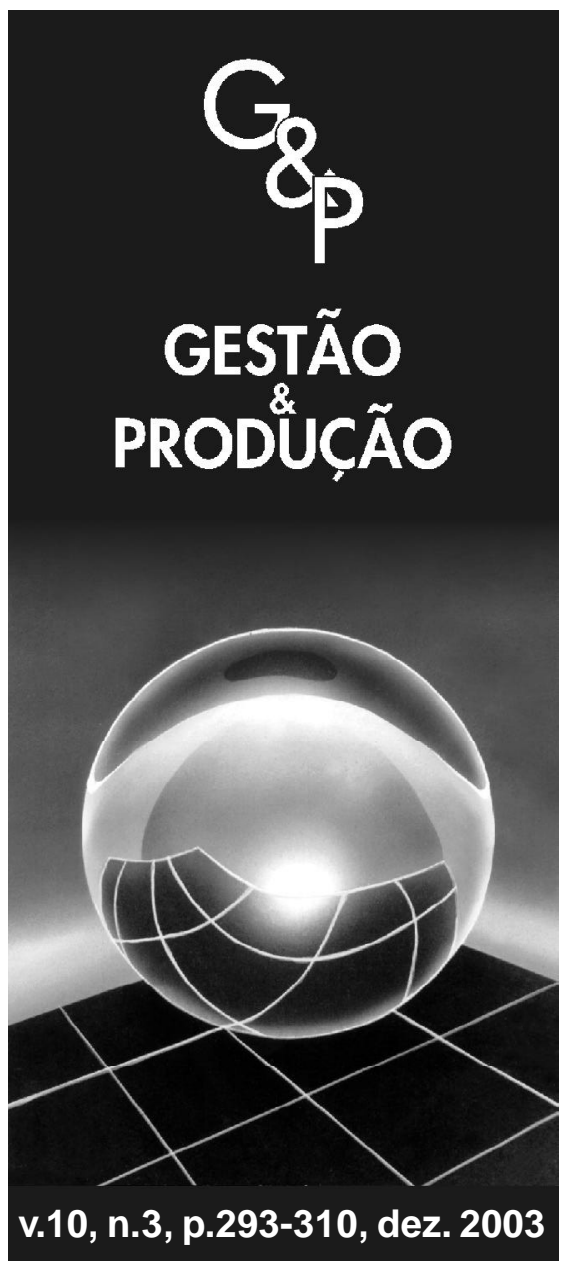

\title{
ESFORÇO TECNOLÓGICO E DESEMPENHO INOVADOR DAS EMPRESAS DO SETOR MÉDICO-HOSPITALAR LOCALIZADAS EM SÃO CARLOS, SP
}

\author{
Denise Luciana Rieg (bolsista FAPESP) \\ Alceu Gomes Alves Filho \\ Departamento de Engenharia de Produção, \\ Universidade Federal de São Carlos, \\ C.P. 676, CEP 13565-905, São Carlos, SP, \\ e-mails: riegsc@yahoo.com, \\ alceu@power.ufscar.br
}

Recebido em 24/6/2003

Aceito em 28/11/2003

\section{Resumo}

O presente artigo reúne informações sobre os esforços tecnológicos e o desempenho inovador de 12 empresas do setor de equipamentos médico-hospitalares localizadas no município de São Carlos, SP. As informações foram obtidas por meio de entrevistas realizadas com diretores ou proprietários dessas empresas, que responderam a um conjunto de questões estruturadas, preparadas para tal finalidade. Nossas análises dessas informações indicam que tais empresas vêm despendendo esforços tecnológicos expressivos, direcionados tanto a atividades internas de P\&D-em maior proporção - quanto à aquisição de tecnologias a partir de fontes externas. As empresas, em sua maioria, não investem em aquisição de tecnologias a partir de fontes externas mais do que um terço do investimento que realizam em $P \& D$ interno. As atividades de $P \& D$ voltam-se principalmente para inovações em produto. Em relação ao desempenho inovador, constata-se que o número de produtos melhorados tecnologicamente supera bastante o número de produtos tecnologicamente novos lançados no mercado por essas empresas nos últimos três anos.

Palavras-chave: inovação, desempenho, setor de equipamentos médico-hospitalares. 


\section{Introdução}

$\mathrm{P}$ rocuramos apresentar neste artigo uma análise de um conjunto de informações sobre esforços tecnológicos e desempenho inovador coletados em empresas do setor médicohospitalar localizadas no município de São Carlos. Essas informações constituem uma base para compreendermos as estratégias tecnológicas adotadas pelas empresas, entendidas aqui como o conjunto de decisões tomadas em relação à acumulação, ao desenvolvimento e ao uso de capacitações e recursos tecnológicos (Zahra, 1996a).

Dessa forma, procuramos sintetizar alguns resultados ainda preliminares do projeto de pesquisa "Estratégia Tecnológica e Competitividade: o caso do setor médico-hospitalar das regiões de São Carlos e Ribeirão Preto", financiado pela Fapesp (Fundação de Amparo à Pesquisa do Estado de São Paulo), que visa a identificar as estratégias tecnológicas adotadas pelas empresas do setor médico-hospitalar localizadas nas regiões de São Carlos e Ribeirão Preto, bem como analisar como tais estratégias contribuem para que essas empresas sejam mais ou menos competitivas.

$\mathrm{O}$ interesse pelas empresas de pequeno porte do setor de equipamentos médico-hospitalares decorre de resultados provenientes de outra pesquisa, intitulada "Caracterização do Perfil da Pequena Empresa de Base Tecnológica no Estado de São Paulo" (Fernandes \& Côrtes, 1998). Consta na base de dados dessa pesquisa que as empresas de base tecnológica (EBTs) paulistas se encontram altamente concentradas em dois setores da Classificação Nacional de Atividades Econômicas (CNAE): equipamentos médicohospitalares e instrumentos de precisão e automatização $(36,0 \%)$ e informática $(22,8 \%)$, correspondendo a 58,8\% das empresas da amostra. Em relação à especialização regional, o setor de equipamentos médicos e de instrumentos de precisão e automação é o primeiro ou o segundo mais importante em todas as regiões estudadas (região metropolitana de São Paulo, Campinas, São José dos Campos, São Carlos e Ribeirão Preto). Nas regiões de São Carlos e Ribeirão Preto, fazem parte desse setor, respectivamente, $41,2 \%$ e 81,8\% das EBTs, sugerindo um nível de especialização não observado nas demais regiões.

Para apresentação dos resultados preliminares da pesquisa, por ora abrangendo apenas as empresas localizadas em São Carlos, expomos a seguir, na Seção 2, os conceitos e os indicadores sobre esforço tecnológico e desempenho inovador utilizados no presente trabalho. Fazemos, depois, um breve apanhado das características gerais do setor médico-hospitalar e do parque industrial da Cidade de São Carlos. Na Seção 4, discorremos sobre o método aplicado para desenvolvimento da pesquisa e, em seguida (Seção 5), mostramos os resultados obtidos quanto aos esforços tecnológicos e ao desempenho inovador das empresas do setor de equipamentos médico-hospitalares de São Carlos. Este artigo é finalizado com breve discussão sobre os resultados obtidos.

\section{Esforço tecnológico e desempenho inovador}

O esforço tecnológico de uma dada empresa pode ser medido pelo montante de recursos que investe em atividades de $\mathrm{P} \& \mathrm{D}$ e em capacitação tecnológica (SOBEET, 2000). De acordo com a SOBEET (2000), a capacitação tecnológica inclui as despesas com suporte e apoio tecnológico a P\&D, como registro de marcas e patentes, treinamento de pesquisadores e manutenção de equipamentos usados em $\mathrm{P} \& \mathrm{D}$; despesas com aquisição de tecnologias a partir de fontes externas; e despesas com engenharia não-rotineira. Esta última inclui projetos de ferramentas e novos produtos e processos produtivos, e de programas de qualidade total, excluindo as atividades de engenharia com baixo teor inovador e de caráter repetitivo.

Especificamente em relação às atividades de P\&D, Wilbon (1999) ressalta a importância de as empresas realizarem tanto pesquisa básica quanto aplicada. A pesquisa básica volta-se a descobertas científicas que possam trazer ganhos para a empresa a longo prazo, enquanto a pesquisa aplicada está orientada ao desenvolvimento de produtos ou processos que possam ser comercializados imediatamente ou em um curto 
espaço de tempo pela empresa. De acordo com Zahra (1996b), em razão do grande investimento associado à pesquisa básica e à necessidade de retorno imediato a partir das descobertas, as novas empresas tendem a se dedicar mais à pesquisa aplicada.

As empresas que fazem pesquisa básica (além da aplicada) geralmente são de grande porte. As pequenas empresas, além de se voltarem preferencialmente à pesquisa aplicada, muitas vezes não possuem departamento estruturado de $\mathrm{P} \& \mathrm{D}$. As atividades de $P \& D$ nas pequenas empresas são realizadas esporadicamente, por diferentes grupos de pessoas, e, geralmente, são desenvolvidas para tentar atender a necessidades imediatas dos clientes. Muitas vezes, também, as atividades de P\&D são realizadas para complementar processos de aquisição de tecnologia de fontes externas às empresas adquirentes. Por exemplo, a empresa pode comprar alguma tecnologia que precisará ser decodificada e incorporada, e as atividades de P\&D são levadas a cabo com tal finalidade. As atividades de $P \& D$ realizadas esporadicamente são denominadas "P\&D semi-estruturado".

Já as fontes externas de tecnologia podem fornecer às empresas acesso um amplo conjunto de capacidades tecnológicas, necessárias ao desenvolvimento de novos produtos e processos ou ao aperfeiçoamento de produtos e processos já existentes, compensando "fraquezas" das atividades de $\mathrm{P} \& \mathrm{D}$ e acelerando o desenvolvimento e a comercialização de novos produtos (Zahra \& Bogner, 1999).

Como expõem Lancot \& Swan (2000), as empresas têm procurado ampliar os recursos tecnológicos dirigidos ao desenvolvimento de produtos e processos novos ou melhorados, mas têm sido freqüentemente incapazes de orientar, ao mesmo tempo e consistentemente, investimentos expressivos e um conjunto complexo de recursos e competências para tal finalidade. Por isso, e também porque os resultados das atividades internas de P\&D são, em geral, incertos (Zahra, 1996b), buscam freqüentemente fontes externas de tecnologia.

Essas fontes externas de tecnologia englobam licenciamentos, alianças estratégicas, compra de tecnologias e contratação de outras empresas, universidades e centros de pesquisa.

Licenciamento é um acordo entre duas partes, uma das quais tem direito de propriedade sobre tecnologia, processo ou informação, enquanto a outra parte quer fazer uso da mesma. Essa segunda parte, então, paga royalties ou alguma outra soma específica em dinheiro ao licenciador em troca da permissão de uso. Geralmente, o objeto de licenciamento está protegido por uma patente e pode ser copiado com a concessão do licenciamento.

As especificações de um licenciamento podem variar. Por exemplo, no caso de licenciamento de um produto patenteado, a autorização pode ser dada somente para sua comercialização, continuando o proprietário do produto a manufaturá-lo. Ou a permissão pode ser dada tanto para comercializá-lo quanto para manufaturá-lo.

Aliança estratégica é definida como uma relação de colaboração entre organizações para alcançar uma meta comum que dificilmente seria atingida isoladamente. Alianças estratégicas podem envolver empresas, universidades, organizações não-lucrativas, organizações governamentais e centros de pesquisa (Lambe \& Spekman, 1997).

Finalizando a exposição do que é comumente compreendido como esforço tecnológico, cabe ressaltar a importância de serem contempladas, ainda, além das atividades de $\mathrm{P} \& \mathrm{D}$ realizadas em centros próprios e estruturados de pesquisa, da compra de tecnologia e das alianças formais, os arranjos menos formalizados de $\mathrm{P} \& \mathrm{D}$ (P\&D semi-estruturados) e as articulações diretas, muitas vezes informais, com centros de pesquisa, outras empresas e universidades.

Considerando esse amplo conjunto de esforços como esforços tecnológicos, ficam reconhecidos os problemas comuns às empresas pertencentes a economias periféricas, como a brasileira, que enfrentam dificuldades para ter acesso a conhecimentos, mercados e crédito (Fernandes et al., 2000). Nos países em desenvolvimento, as atividades de inovação estão, em sua maioria, relacionadas à difusão, à adaptação e a melhorias em tecnologias já existentes. 
Geralmente, essas atividades não são desenvolvidas em departamentos de P\&D formalmente estruturados, mas por meio de outros esforços de inovação anteriormente citados (Quadros et al., 1999).

Em relação ao desempenho inovador, são consideradas as inovações tecnológicas de processo e de produto comercialmente viáveis, resultantes dos esforços tecnológicos despendidos pelas empresas. Mais uma vez, levando-se em conta as condições específicas de um país em desenvolvimento como o Brasil, que se encontra há pouco mais de uma década livre de generalizada proteção comercial (Suzigan, 1992), as inovações tecnológicas de produto e processo a serem consideradas devem incluir tanto as de natureza significativa como as de natureza incremental.

Na presente pesquisa, são utilizadas as definições de inovação de produto e processo, de natureza significativa e incremental, do questionário aplicado na Pesquisa da Atividade Econômica Paulista (PAEP) desenvolvida pela Fundação Sistema Estadual de Análise de Dados (SEADE), conforme apresentamos a seguir.

Inovações de produto de natureza significativa referem-se a produtos inteiramente novos, os quais apresentam características tecnológicas ou de uso e finalidade que os distinguem daqueles produzidos até então. Por outro lado, inovações de produto de natureza incremental correspondem a substanciais aperfeiçoamentos de produtos previamente existentes (produtos melhorados). Essa mesma classificação pode ser feita para as inovações de processo. Do mesmo modo, inovações de processo de natureza significativa correspondem à incorporação de novos processos de produção, e inovações de natureza incremental correspondem a modificações tecnológicas em processos já adotados pela empresa (SEADE, 1998).

Complementando a compreensão sobre desempenho inovador, adota-se como um de seus indicadores nesta pesquisa, além das quantidades de inovações de produto e de processo de natureza incremental e significativa, a parcela do faturamento derivada de novos produtos ou de produtos melhorados, desenvolvidos internamente à empresa (não-licenciados) e introduzidos no mercado em um período determinado. Segundo a SOBEET (2000), “...o êxito dos investimentos em inovações e capacitação tecnológica pode ser mensurado pela porcentagem do faturamento de vendas gerado por meio de produtos lançados no mercado...", provenientes de tais investimentos.

O Apêndice 1 apresenta o conjunto dos indicadores de esforço tecnológico e desempenho inovador utilizado na presente pesquisa, com base no exposto anteriormente. Eles foram obtidos nas empresas analisadas em um período de três anos, de 2000 a 2002.

\section{3. Âmbito de estudo}

\subsection{O setor médico-hospitalar}

De acordo com a Classificação Nacional das Atividades Econômicas (CNAE), a indústria de equipamentos médico-hospitalares é formada pelas empresas produtoras de aparelhos e instrumentos para uso médico-hospitalar, odontológico e de laboratório e aparelhos ortopédicos. Os fornecedores de componentes para esses equipamentos também entram nessa classificação (CNAE, 1997).

Furtado (1999) apresenta uma caracterização da dimensão internacional da indústria de equipamentos médico-hospitalares. Segundo esse autor, essa indústria representa um mercado global de 105 bilhões de dólares, com ritmo de crescimento anual de $2,5 \%$ nos últimos anos. No Brasil, as empresas do referido setor apresentaram faturamento de 3,5 bilhões de reais em 2000 (Telles, 2002).

O desenvolvimento tecnológico na indústria de equipamentos médico-hospitalares apresenta ritmo bastante acelerado, apoiado em múltiplas fontes de insumos. Os avanços científicos e tecnológicos, e as inovações de setores como mecânica de precisão, eletrônica digital, informática e química dão suporte ao dinamismo tecnológico do setor médico-hospitalar, que, por sua vez, fortalece a oferta da indústria de equipamentos 
médico-hospitalares (Furtado \& Souza, 2000). Deve-se considerar também que a baixa elasticidade de substituição dos produtos constitui outro fator que amplia a oferta dessa indústria, promovendo o desenvolvimento de soluções diferenciadas e hiperespecializadas por parte das empresas (Furtado, 1999).

Segundo a Associação Brasileira da Indústria Médico-Odontológica (ABIMO), no Brasil, o setor médico-hospitalar é composto por cerca de 500 empresas. A maioria delas é de pequeno porte, $68 \%$; $28 \%$ são de médio porte; e apenas $3,3 \%$ das empresas são de grande porte (Guia de Fornecedores Hospitalares, 2000). Cabe ressaltar que a classificação do setor utilizada pela $A B I M O$ é diferente daquela usada pela CNAE, ou seja, a ABIMO também inclui no setor médico-hospitalar as empresas produtoras de materiais de consumo, como as que produzem agulhas e seringas, algodão e gazes, categutes, sondas e catéteres, luvas cirúrgicas, reagentes, etc. No presente trabalho, utilizamos a classificação definida pela CNAE.

Outros dados também obtidos pela ABIMO revelam que as empresas localizadas no território nacional são predominantemente de capital nacional, correspondendo a 79,64\% das empresas. Apenas 20,36\% são de capital estrangeiro.

A produção de equipamentos visa a atender a demanda do setor privado ( $48 \%$ ) e do governo $(43,3 \%)$. Uma parcela muito pequena da produção é destinada a exportações.

Por fim, cabe observar que $80 \%$ das empresas brasileiras do setor médico-hospitalar estão instaladas no Estado de São Paulo (Telles, 2002).

\subsection{A estrutura institucional a a indústria no município de São Carlos}

O município de São Carlos vem sendo caracterizado como pólo tecnológico, por abrigar ampla estrutura institucional em ciência e tecnologia e um conjunto relativamente diversificado de empresas industriais. Estão instalados no município, já há bastante tempo, um campus da Universidade de São Paulo (USP) e um da Universidade Federal de São Carlos (UFSCar). Em ambos os campi as áreas de ciências exatas e engenharias são bastante desenvolvidas. Encontram-se também em São Carlos dois centros de pesquisa da Empresa Brasileira de Agropecuária (EMBRAPA): o Centro Nacional de Pesquisa e Desenvolvimento de Instrumentos Agropecuários (CNPDIA) e o Centro de Pesquisa de Pecuária do Sudeste (CPPSE).

Em 1984, com o intuito de promover o desenvolvimento do pólo tecnológico, especialmente das pequenas empresas de alta tecnologia criadas a partir de pesquisas geradas nas instituições de ensino e pesquisa e nas empresas nascentes, foi instalada no município a Fundação Parque de Alta Tecnologia (ParqTec). Dois centros incubadores de empresas estão vinculados à ParqTec: o Centro Incubador de Empresas Tecnológicas (CINET) e o Centro Incubador de Empresas de Software (SOFTNET) (Torkomian, 1997).

O setor industrial de São Carlos abrange, atualmente, cerca de 600 empresas, grande parte delas de pequeno e médio porte, em diversos setores, mas com predominância do segmento metal-mecânico. Algumas das poucas empresas de grande porte, entretanto, fornecem uma visão mais rápida da diversificação e da complexidade da tecnologia empregada nas empresas do município: Volkswagen (motores), Tecumseh (compressores), Eletrolux (geladeiras e fogões), FaberCastell (lápis), Toalhas São Carlos (toalhas). Outras informações podem ser obtidas no site <http://www.saocarlos.sp.gov.br>.

Fazem parte do conjunto de empresas de pequeno porte de São Carlos aquelas do setor de equipamentos médico-hospitalares estudadas neste trabalho, a maioria considerada de base tecnológica. Como apresentado na introdução deste trabalho, a pesquisa desenvolvida por Fernandes \& Côrtes (1998) mostra que 41,2\% das empresas de base tecnológica de São Carlos se concentram no referido setor. E as regiões de São Carlos e de Ribeirão Preto apresentam um nível de especialização muito alto, não observado em nenhuma das demais regiões paulistas.

Nesse contexto institucional, que pode ser considerado propício ao desenvolvimento de atividades inovadoras, procuramos caracterizar os 
esforços tecnológicos e o desempenho inovador das empresas do setor de equipamentos médicohospitalares.

\section{Método}

Primeiramente, buscou-se identificar as empresas do setor médico-hospitalar localizadas em São Carlos. Como não pudemos encontrar qualquer documento que apresentasse as empresas do município agrupadas por setor de atividades (o que dificultou bastante a identificação das empresas do setor), obtivemos primeiro, na Prefeitura de São Carlos, o "Relatório de Empresas por Atividade Municipal". Nesse documento figuram apenas os nomes das empresas localizadas em São Carlos (aproximadamente 600) e seus respectivos endereços. Em seguida, após obtermos os telefones dessas empresas (via lista telefônica por endereços), entramos em contato com as mesmas para verificar quais delas eram produtoras de equipamentos ou de instrumentos médicos ou odontológicos. Dessa forma chegamos a um total de 15 empresas.

O meio utilizado para conduzir a pesquisa foi a aplicação de um questionário estruturado com respostas circunscritas a uma escala de cinco pontos (Apêndice 2), de maneira a aumentar a objetividade da coleta de dados e, conseqüentemente, facilitar o processo de análise do esforço tecnológico despendido por essas empresas. Em outras palavras, as respostas dos entrevistados foram convertidas, durante a aplicação do questionário (aos entrevistados), em categorias expressas numericamente, o que permite que os dados coletados sejam contados e tabulados.

Para a construção desse questionário foram levantados indicadores múltiplos para o esforço tecnológico e desempenho inovador (Apêndice 1 ), de modo que se pudesse verificar a consistência das informações obtidas com os entrevistados sobre os diversos indicadores.

Das 15 empresas do setor médico-hospitalar localizadas em São Carlos, pudemos coletar dados em 12 delas. As outras 3 empresas não se coloca- ram à disposição para responder ao questionário. A pesquisa foi realizada em 2002, sendo os questionários aplicados pessoalmente por um dos autores deste trabalho, de modo a reduzir os possíveis problemas de interpretação sobre os indicadores utilizados.

\section{Resultados}

Nesta seção são apresentados alguns dos resultados obtidos com a aplicação do questionário constante no Apêndice 2 nas 12 empresas do setor médico-hospitalar localizadas no município de São Carlos. Primeiramente, são apresentadas informações gerais sobre essas empresas e, posteriormente, são mostrados os resultados sobre seus esforços tecnológicos e desempenho inovador, referentes aos anos de 2000, 2001 e 2002.

$\mathrm{O}$ primeiro aspecto a ser destacado se refere à atividade econômica predominante dessas empresas. Das 12 empresas estudadas, 5 são produtoras de aparelhos e instrumentos ópticos, 4 são produtoras de aparelhos e instrumentos para uso odontológico, 4 fornecem exclusivamente partes de equipamentos médicos e odontológicos e as 2 empresas restantes produzem equipamentos médicos variados. Todas são inteiramente de capital nacional.

O segundo aspecto importante é o porte das empresas entrevistadas. Para tal classificação adotamos o critério estabelecido pela CIESP (Fernandes \& Côrtes, 1998), segundo o qual empresas com até 29 funcionários são consideradas microempresas; com 30 a 199, pequenas empresas; de 200 a 700, empresas de porte médio; e aquelas com mais de 700 funcionários são consideradas de grande porte. Desse modo, das 12 empresas estudadas, 8 são microempresas, 3 são pequenas empresas e apenas 1 é de médio porte. Não foi identificada nenhuma empresa de grande porte.

$\mathrm{O}$ terceiro aspecto do perfil das empresas a ser observado é sua idade. Das 12 empresas estudadas, 5 têm menos de 5 anos de vida e 
apenas 2 empresas estão há mais de 20 anos no mercado. As 3 empresas que há mais tempo atuam no mercado, há 26,25 e 17 anos, são também aquelas de maior porte, com 40, 30 e 200 funcionários, respectivamente.

Uma vez apresentados os aspectos gerais dessas empresas, passamos à análise dos esforços tecnológicos por elas despendidos.

Primeiramente, são analisadas as atividades internas de $\mathrm{P} \& \mathrm{D}$ realizadas nas 12 empresas estudadas. Como pode ser observado na Tabela 1, apenas duas empresas não possuem departamento de pesquisa e desenvolvimento, três possuem departamentos de $\mathrm{P} \& \mathrm{D}$ estruturados e mais da metade das empresas estudadas possui P\&D semi-estruturado. Cabe lembrar que se entende que as empresas possuem $\mathrm{P} \& \mathrm{D}$ semiestruturado quando as atividades de P\&D são realizadas esporadicamente por diferentes grupos de pessoas dentro da empresa.

Cabe observar também que os departamentos de $\mathrm{P} \& \mathrm{D}$ estruturados e semi-estruturados possuem em média quatro funcionários, sendo a maioria técnicos de nível médio.

Em relação aos gastos com atividades internas de P\&D, observa-se na Tabela 1 que dentre as empresas com P\&D estruturado ou semiestruturado, $50 \%$ investem entre $10 \%$ e $15 \%$ do faturamento em tais atividades. Observa-se também que a maior parte dos gastos é destinada a inovações em produtos. Das dez empresas que realizam $P \& D$, oito dedicam mais de $70 \%$ de seus gastos com $\mathrm{P} \& \mathrm{D}$ a inovações em produtos.

A seguir são analisadas as despesas com aquisição de tecnologias desenvolvidas externamente às empresas. Se comparados os dados referentes aos gastos com aquisição de tecnologias a partir de fontes externas à empresa em relação ao faturamento (Tabela 2) com os gastos com atividades internas de $\mathrm{P} \& \mathrm{D}$ em relação ao faturamento (Tabela 1), constata-se que as empresas vêm investindo mais em $\mathrm{P} \& \mathrm{D}$ interno do que na aquisição de tecnologias a partir de fontes externas. As empresas, em sua maioria, não têm investido em aquisição de tecnologias a partir de fontes externas mais do que um terço do investimento realizado em $\mathrm{P} \& \mathrm{D}$ interno.

Ainda em relação às fontes externas de tecnologia, a Tabela 2 mostra que o recurso menos utilizado pelas empresas é a compra e o licenciamento de novas tecnologias, desenvolvidas por outras empresas para introduzi-las em seus produtos.

Apenas 4 das 12 empresas estudadas realizam esse tipo de aquisição de tecnologias e, mesmo assim, com baixa freqüência: compraram ou licenciaram tecnologias desenvolvidas por terceiros, em média, 2 vezes nos últimos 3 anos.

Tabela 1 - Atividades internas de pesquisa e desenvolvimento (P\&D) nas 12 empresas estudadas (média dos últimos 3 anos).

\begin{tabular}{|c|c|c|c|c|c|c|c|c|c|c|c|c|}
\hline & $\mathbf{E}_{1}$ & $\mathbf{E}_{2}$ & $\mathbf{E}_{3}$ & $\mathbf{E}_{\mathbf{4}}$ & $\mathbf{E}_{5}$ & $\mathbf{E}_{6}$ & $\mathbf{E}_{7}$ & $\mathbf{E}_{8}$ & $\mathbf{E}_{9}$ & $\mathbf{E}_{10}$ & $\mathbf{E}_{11}$ & $\mathbf{E}_{12}$ \\
\hline $\begin{array}{l}\text { Possui P\&D (1) estruturado, (2) semi- } \\
\text { estruturado e (3) não possui P\&D }\end{array}$ & 3 & 1 & 2 & 1 & 2 & 2 & 2 & 3 & 1 & 2 & 2 & 2 \\
\hline Número de engenheiros e cientistas no $\mathrm{P} \& \mathrm{D}$ & 0 & 0 & 2 & 1 & 0 & 0 & 2 & 0 & 5 & 5 & 2 & 3 \\
\hline Número de técnicos de nível médio no P\&D & 0 & 3 & 2 & 1 & 3 & 4 & 1 & 0 & 6 & 3 & 1 & 2 \\
\hline $\begin{array}{l}\text { Gastos com P\&D interno em relação ao } \\
\text { faturamento }(\%)\end{array}$ & 0 & 5 & 15 & 10 & 5 & 15 & 5 & 0 & 15 & 6 & 10 & 10 \\
\hline $\begin{array}{l}\% \text { das despesas em } \mathrm{P} \& \mathrm{D} \text { destinadas a } \\
\text { inovações em produtos }\end{array}$ & 0 & 70 & 100 & 100 & 50 & 67 & 100 & 0 & 80 & 90 & 100 & 70 \\
\hline $\begin{array}{l}\% \text { das despesas em P\&D destinadas a } \\
\text { inovações em processos }\end{array}$ & 0 & 30 & 0 & 0 & 50 & 33 & 0 & 0 & 20 & 10 & 0 & 30 \\
\hline
\end{tabular}


Ainda na Tabela 2, observa-se que a contratação de outras empresas, universidades ou centros de pesquisa para desenvolver tecnologias de produto e processo é praticada mais freqüentemente pelas empresas estudadas do que a compra e o licenciamento de tecnologias. Das 12 empresas, sete afirmaram realizar esse tipo de contratação. Dessas 7, 4 contratam diferentes instituições para desenvolvimento de tecnologias no mínimo 2 vezes ao ano, ao passo que as outras 3 empresas contrataram outras instituições para desenvolver tecnologias no máximo 2 vezes nos últimos 3 anos.

A aliança com centros de pesquisa, universidades e outras empresas, a fim de desenvolver produtos e processos tecnologicamente novos ou melhorados, vem sendo a atividade praticada com maior frequiência pelas empresas estudadas. Como mostra a Tabela 2, das 12 empresas analisadas, apenas 3 não realizam esse tipo de atividade. A maioria das empresas (7) faz alianças estratégicas para desenvolver produtos e processos tecnolo- gicamente novos ou melhorados no mínimo 2 vezes ao ano. A Tabela 3 apresenta os dados referentes à participação de diferentes instituições nas empresas estudadas: em alianças estratégicas, para desenvolver tecnologias novas ou fazer melhorias nas tecnologias existentes, direcionadas aos produtos ou aos processos de produção. Cabe destacar que são alianças estratégicas informais, em sua maioria.

Nas alianças estratégicas para desenvolver produtos tecnologicamente novos ou melhorados, os clientes e os fornecedores das empresas aparecem como os principais parceiros. Das 12 empresas estudadas, 9 fazem alianças estratégicas com seus clientes e fornecedores. Cabe ressaltar, ainda, que as empresas fazem alianças mais freqüentemente com os clientes do que com os fornecedores. Enquanto 5 das 9 empresas que realizam alianças estratégicas com os seus clientes o fazem no mínimo duas vezes ao ano, 6 das 9 empresas que realizam alianças com os seus fornecedores o fazem uma vez ao ano.

Tabela 2 - Fontes externas de aquisição de tecnologias (média dos últimos três anos).

\begin{tabular}{|c|c|c|c|c|c|c|c|c|c|c|c|c|}
\hline & $\mathbf{E}_{1}$ & $\mathbf{E}_{2}$ & $\mathbf{E}_{\mathbf{3}}$ & $\mathbf{E}_{4}$ & $\mathbf{E}_{5}$ & $\mathbf{E}_{6}$ & $\mathbf{E}_{7}$ & $\mathbf{E}_{8}$ & $\mathbf{E}_{9}$ & $\mathbf{E}_{10}$ & $\mathbf{E}_{11}$ & $\mathbf{E}_{12}$ \\
\hline $\begin{array}{l}\text { Gastos com aquisição de tecnologias de } \\
\text { produto e processo a partir de fontes } \\
\text { externas à empresa em relação ao } \\
\text { faturamento }(\%)\end{array}$ & 0 & 1,5 & 5 & 0 & 1 & 10 & 2 & 0 & 5 & 3 & 1 & 10 \\
\hline $\begin{array}{l}\text { Freqüência com que a empresa compra } \\
\text { ou licencia novas tecnologias } \\
\text { desenvolvidas por outras empresas }\end{array}$ & NA & NA & NA & NA & $\mathrm{MB}$ & NA & $\mathrm{M}$ & NA & B & NA & NA & $\mathrm{MB}$ \\
\hline $\begin{array}{l}\text { Freqüência com que a empresa contrata } \\
\text { outras empresas, universidades ou } \\
\text { centros de pesquisa para desenvolver } \\
\text { tecnologias de produto e processo }\end{array}$ & NA & A & NA & NA & B & $\mathrm{MB}$ & A & NA & NA & A & B & A \\
\hline $\begin{array}{l}\text { Freqüência com que a empresa faz } \\
\text { alianças estratégicas com centros de } \\
\text { pesquisa, universidades e outras } \\
\text { empresas para desenvolver produtos e } \\
\text { processos tecnologicamente novos ou } \\
\text { melhorados }\end{array}$ & $\mathrm{M}$ & A & $\mathrm{M}$ & NA & MA & A & NA & NA & A & MA & A & A \\
\hline
\end{tabular}

*NA = não se aplica à empresa; MB (muito baixa) = uma vez nos últimos três anos; B (baixa) = duas vezes nos últimos três anos; $\mathrm{M}$ (média) = uma vez por ano; $\mathrm{A}$ (alta) = duas vezes ao ano; MA (muito alta) = a empresa faz isso constantemente. 
Em seguida, os centros de pesquisa e as universidades aparecem como as instituições que mais participam com as empresas estudadas das alianças estratégicas para desenvolvimento de novos produtos. Quatro das doze empresas afirmaram realizar alianças com essas instituições no mínimo duas vezes ao ano. Por outro lado, a procura por outras empresas nacionais (que não sejam fornecedores ou clientes das empresas) e internacionais para realizar alianças desse tipo praticamente não ocorreu nessas empresas nos últimos três anos (linhas 4 e 5 da Tabela 3).

Quanto às alianças estratégicas para desenvolver processos tecnologicamente novos ou melhorados, os fornecedores aparecem destacadamente como os principais parceiros. Mesmo assim, a freqüência com que essas alianças ocorrem é relativamente baixa: em média não mais do que duas vezes nos últimos três anos. Apenas três empresas, em média, mantiveram alianças com universidades, centros de pesquisa, clientes e outras empresas nacionais, não revelando, portanto, participação quantitativamente importante para o conjunto das empresas.
Finalmente, os dados da Tabela 3 mostram que alianças estratégicas para o desenvolvimento de processos novos ou melhorados ocorreram com empresas estrangeiras em apenas um caso e, mesmo assim, com baixa freqüência.

Concluindo a análise dos esforços tecnológicos despendidos pelas empresas estudadas, têm-se as despesas com patentes em relação às vendas (ou faturamento) das empresas. As 12 empresas investigadas gastaram, em média, nos últimos três anos, não mais do que $3 \%$ com patentes em relação ao faturamento.

Sumarizando, os esforços tecnológicos das empresas estudadas concentram-se nas atividades internas de $\mathrm{P} \& \mathrm{D}$, voltadas tanto para produto, principalmente, quanto para processo. Quando a busca por tecnologia ocorre fora da empresa, o principal mecanismo utilizado é a aliança estratégica informal com clientes e fornecedores. Pouco é investido em patentes para proteção dos novos produtos. A seguir, são apresentados resultados referentes ao desempenho inovador das 12 empresas do setor médico-hospitalar localizadas em São Carlos.

Tabela 3 - Alianças estratégicas para desenvolver produtos e processos tecnologicamente novos ou melhorados.

\begin{tabular}{|c|c|c|c|c|c|c|c|c|c|c|c|c|c|}
\hline & & $\mathbf{E}_{1}$ & $\mathbf{E}_{2}$ & $\mathbf{E}_{3}$ & $\mathbf{E}_{\mathbf{4}}$ & $\mathbf{E}_{5}$ & $E_{6}$ & $\mathbf{E}_{7}$ & $\mathbf{E}_{8}$ & $E_{9}$ & $\mathbf{E}_{10}$ & $E_{11}$ & $E_{12}$ \\
\hline \multirow{2}{*}{$\begin{array}{l}\text { Frequiência com que a } \\
\text { empresa faz alianças com } \\
\text { centros de pesquisa e } \\
\text { universidades }\end{array}$} & Produto & NA & A & NA & NA & A & NA & NA & NA & MB & MA & NA & A \\
\hline & Proc & NA & M & NA & NA & NA & NA & NA & NA & NA & A & A & A \\
\hline \multirow{2}{*}{$\begin{array}{l}\text { Frequiência com que a } \\
\text { empresa faz alianças com } \\
\text { fornecedores }\end{array}$} & Prod & M & M & M & NA & M & M & MB & NA & NA & B & B & M \\
\hline & Proces & B & B & MB & NA & B & M & NA & NA & NA & M & B & M \\
\hline \multirow{2}{*}{$\begin{array}{l}\text { Frequiência com que a } \\
\text { empresa faz alianças com } \\
\text { clientes }\end{array}$} & Produto & B & M & M & NA & MA & A & NA & NA & A & MA & A & $\mathbf{I N}$ \\
\hline & Proce & NA & NA & NA & NA & NA & A & NA & NA & NA & NA & M & $\mathrm{M}$ \\
\hline \multirow{2}{*}{$\begin{array}{l}\text { Freqüência com que a } \\
\text { empresa faz alianças com } \\
\text { outras empresas nacionais }\end{array}$} & & $\mathrm{N}$ & M & NA & NA & MB & D & NA & NA & NA & NA & NA & A \\
\hline & Processo & NA & M & NA & NA & NA & NA & NA & NA & NA & B & NA & $\mathbf{A}$ \\
\hline \multirow{2}{*}{$\begin{array}{l}\text { Freqüência com que a } \\
\text { empresa faz alianças com } \\
\text { empresas estrangeiras }\end{array}$} & Produ & NA & NA & NA & NA & M & NA & NA & NA & NA & NA & NA & 3 \\
\hline & Processo & NA & NA & NA & NA & NA & NA & NA & NA & NA & NA & NA & B \\
\hline
\end{tabular}

*NA = não se aplica à empresa; $\mathrm{MB}$ (muito baixa) $=1$ vez nos últimos 3 anos; $\mathrm{B}$ (baixa) $=2$ vezes nos últimos 3 anos; $\mathrm{M}$ $($ média $)=1$ vez por ano; $\mathrm{A}($ alta $)=2$ vezes ao ano; MA (muito alta $)=$ a empresa faz isso constantemente. 
A Tabela 4 apresenta os dados referentes às inovações de produto e processo. Como exposto na Seção 2, as inovações de produto correspondem à introdução de novos produtos na linha de produção da empresa ou às modificações tecnológicas de produtos já existentes, mas excluem inovações puramente estéticas ou de estilo. Nessa mesma linha, inovações em processo correspondem à introdução de novos processos produtivos na empresa ou às modificações tecnológicas de processos já existentes.

Diante disso, pode ser observado na Tabela 4 que 5 das 12 empresas estudadas lançaram no mercado pelo menos 6 produtos novos nos últimos 3 anos; 2 não lançaram produtos novos; e as 5 empresas restantes lançaram de 2 a 4 produtos novos. Em relação às inovações em produto de natureza incremental, observa-se que 2 empresas se destacam com mais de 10 produtos desse tipo lançados no mercado nos últimos 3 anos; 5 empresas lançaram entre 5 e 10 produtos; e 4 lançaram menos de 5 produtos melhorados tecnologicamente.

Cabe ressaltar que as duas empresas que se destacaram no lançamento de produtos melhorados tecnologicamente possuem $\mathrm{P} \& \mathrm{D}$ estru- turado. Essa relação não pode ser observada quando são focalizados produtos novos.

Quanto às inovações em processo, observase que as inovações tanto do tipo significativa quanto incremental ocorrem freqüentemente na maioria das empresas estudadas, em média três vezes ao ano.

Finalizando a análise do desempenho inovador, encontram-se na Tabela 4 as parcelas do faturamento derivadas de novos produtos ou de produtos melhorados desenvolvidos internamente às empresas e introduzidos no mercado nos últimos três anos. Os resultados mostram que as empresas do setor médico-hospitalar de São Carlos alcançaram, segundo esse critério, excelente desempenho: para oito das dez empresas que inovam, mais de $50 \%$ do faturamento presente é fruto do lançamento de produtos novos ou melhorados tecnologicamente. Tal desempenho se deve à necessidade de alterações tecnológicas constantes nos produtos finais, como já comentado na caracterização do setor médico-hospitalar, que apresenta alto dinamismo tecnológico, alimentado pelos avanços científicos e tecnológicos e pelas inovações de setores correlacionados.

Tabela 4 - Inovações em produto e processo nos últimos três anos.

\begin{tabular}{|c|c|c|c|c|c|c|c|c|c|c|c|c|}
\hline & $\mathbf{E}_{1}$ & $\mathbf{E}_{2}$ & $\mathbf{E}_{3}$ & $\mathbf{E}_{4}$ & $\mathbf{E}_{5}$ & $\mathbf{E}_{6}$ & $\mathbf{E}_{7}$ & $\mathbf{E}_{8}$ & $\mathbf{E}_{9}$ & $\mathbf{E}_{10}$ & $\mathbf{E}_{11}$ & $\mathbf{E}_{12}$ \\
\hline $\begin{array}{l}\text { Número aproximado de inovações de } \\
\text { produtos de natureza significativa }\end{array}$ & 0 & 8 & 2 & 9 & 4 & 4 & 13 & 0 & 3 & 6 & 6 & 2 \\
\hline $\begin{array}{l}\text { Número aproximado de inovações de } \\
\text { produtos de natureza incremental }\end{array}$ & 1 & 13 & 2 & 6 & 2 & 5 & 4 & 0 & 20 & 9 & 6 & 5 \\
\hline Número de patentes de produto & 0 & 4 & 0 & 4 & 0 & 0 & 0 & 0 & 10 & 4 & 0 & 3 \\
\hline $\begin{array}{l}\text { Número aproximado de inovações de } \\
\text { processo de natureza significativa }\end{array}$ & 0 & 0 & 9 & 0 & 9 & 9 & 0 & 0 & 9 & 9 & 0 & 3 \\
\hline $\begin{array}{l}\text { Número aproximado de inovações de } \\
\text { processo de natureza incremental }\end{array}$ & 0 & IC & IC & 0 & 9 & IC & 9 & 0 & 9 & IC & 0 & 3 \\
\hline $\begin{array}{l}\text { Parcela do faturamento derivada de } \\
\text { novos produtos ou produtos melhorados } \\
\text { introduzidos no mercado nos últimos } \\
\text { três anos }\end{array}$ & 0 & 100 & 27 & 100 & 68 & 40 & 90 & 0 & 50 & 90 & 25 & 60 \\
\hline
\end{tabular}




\section{Considerações finais}

O objetivo deste artigo foi apresentar o conjunto dos esforços tecnológicos e dos desempenhos inovadores das empresas do setor médicohospitalar localizadas em São Carlos, SP. Para tal, foi realizado um estudo empírico com 12 empresas (dentre as 15 do referido setor presentes no município). A pesquisa mostrou, por meio dos dados coletados, os principais aspectos dos esforços tecnológicos despendidos e dos desempenhos inovadores obtidos por essas empresas.

Pudemos constatar que os esforços tecnológicos das empresas estudadas se concentram nas atividades internas de $\mathrm{P} \& \mathrm{D}$, voltados principalmente para desenvolvimento de novos produtos. Das dez empresas que realizam P\&D, oito dedicam mais de $70 \%$ de seus gastos com $P \& D$ a inovações em produtos.

Essa concentração do esforço tecnológico nas atividades de $\mathrm{P} \& \mathrm{D}$ para desenvolvimento de produtos se diferencia do comportamento geral das empresas industriais no Estado de São Paulo (Quadros et al., 1999). Esses autores, ao analisarem a base de dados da PAEP, constatam que, para mais de 67,5\% das empresas industriais paulistas, as inovações entre 1994-96 tiveram origem principalmente em suas relações com clientes. As atividades de P\&D aparecem em quarto lugar entre os insumos necessários para o desenvolvimento de produtos e processos novos e melhorados tecnologicamente. Se ainda forem consideradas na análise apenas as empresas de pequeno porte (como no caso das empresas aqui analisadas), as atividades de $\mathrm{P} \& \mathrm{D}$ aparecem também em quarto lugar como fonte de insumos mais importante. Como exposto pelos autores, esses resultados revelam um comportamento de inovação predominantemente defensivo, ou seja, as empresas se esforçam para suprir as necessidades do mercado (market pull), mas não para antecipar essas necessidades e explorar oportunidades tecnológicas (technology push).

Este último comportamento, mais ofensivo, seria, então, aquele que se esperaria das empresas que têm por principal fonte de inovação as atividades internas de P\&D, como as empresas estu- dadas. Entretanto, não é isso que se verifica nas empresas aqui analisadas, como explicado a seguir.

Embora essa ênfase em P\&D interno pudesse gerar a expectativa de encontrar um número relativamente alto de inovações de produtos de natureza significativa nessas empresas, não foi o que pudemos observar. O montante de inovações de produto de natureza incremental é superior ao montante de inovações de produto de natureza significativa. Observadas caso a caso, entretanto, em três empresas o número de inovações de produto de natureza significativa foi maior do que o número de inovações de produto de natureza incremental (Tabela 4, empresas $\mathrm{E}_{4}, \mathrm{E}_{5}, \mathrm{E}_{7}$ ).

Esses dados revelam que, a despeito da ênfase em atividades internas de $\mathrm{P} \& \mathrm{D}$, a maioria das empresas segue preferencialmente (mas não totalmente) o padrão de suprir as necessidades tecnológicas de mercado, o que implica o desenvolvimento de inovações de produto de natureza incremental como comportamento dominante.

Outros dados que vão ao encontro dessa observação são aqueles referentes à estruturação dos departamentos de $\mathrm{P} \& \mathrm{D}$ das empresas investigadas. Esses departamentos são informalmente organizados na maioria das empresas, ou seja, 58\% possuem P\&D semi-estruturado. Apenas 25\% possuem $\mathrm{P} \& \mathrm{D}$ estruturado. Como explicado anteriormente, as atividades esporádicas de $\mathrm{P} \& \mathrm{D}$ geralmente são realizadas para atender a necessidades do mercado. Muitas vezes, também, as atividades de P\&D são realizadas para complementar algum processo de aquisição de tecnologia a partir de fontes externas à empresa. Entretanto, sabe-se que as empresas estudadas fazem pouco uso dos mecanismos de compra e licenciamento de tecnologias desenvolvidas por terceiros.

Mas observa-se que, comparado aos demais setores no Brasil, esse conjunto de empresas despende esforços tecnológicos significativos. Apenas como referência, enquanto nas empresas aqui pesquisadas a média de investimentos em $\mathrm{P} \& \mathrm{D}$ em relação ao faturamento é de $8 \%$, nas subsidiárias das multinacionais no Brasil, instaladas em setores relativamente dinâmicos, esse índice, segundo a SOBEET (2000), é igual a apenas $2,14 \%$. 
Nota-se que, quanto à estruturação dos setores de $\mathrm{P} \& \mathrm{D}$, o comportamento das empresas aqui analisadas é semelhante, quantitativamente, ao encontrado na pesquisa de Fernandes et al. (2000), cujos dados abrangem as empresas de base tecnológicas do Estado de São Paulo. Para as empresas do Estado no setor médico-hospitalar, a referida pesquisa mostrou que $26,5 \%$ delas possuem $P \& D$ estruturado e que $66,7 \%$ realizam atividades de $\mathrm{P} \& \mathrm{D}$, mas não de modo sistemático e contínuo. Considerados todos os 12 setores analisados, vários deles destacados na literatura como potencialmente inovadores (Pavitt, 1984), os valores percentuais não são muito diferentes: $22,1 \%$ das empresas estudadas possuem $\mathrm{P} \& \mathrm{D}$ estruturado, $72,8 \%$ possuem $\mathrm{P} \& \mathrm{D}$ semi-estruturado e apenas $5,1 \%$ não realizam atividade interna de pesquisa e desenvolvimento.

Portanto, a maioria das empresas do setor médico-hospitalar localizadas em São Carlos possui estrutura de P\&D informalmente organizada, seguindo o comportamento geral das empresas de base tecnológica do Estado de São Paulo. Esse comportamento, como já exposto, revela que as empresas realizam preferencialmente atividades de inovação relacionadas à difusão, à adaptação e a melhorias em tecnologias já existentes, atividades típicas de países em desenvolvimento.

Finalizando as observações em relação aos investimentos em $\mathrm{P} \& \mathrm{D}$ realizados pelas empresas estudadas, não foi observada relação entre idade, tamanho das empresas e intensidade dos investimentos em P\&D.

Também foi constatado que a maioria das empresas estudadas não tem investido em aquisição de tecnologia a partir de fontes externas mais do que um terço do investimento que realizam em P\&D interno. Quando a busca por tecnologia se dá fora da empresa, o principal mecanismo utilizado é a aliança estratégica informal.

Os principais parceiros nessas alianças são clientes e fornecedores, parcerias bastante coerentes com o padrão de suprir as necessidades tecnológicas de mercado adotado pelas empresas. Em seguida, as universidades e os centros de pesquisa aparecem como as instituições que mais participam de alianças estratégicas com as empresas estudadas para desenvolvimento de novos produtos. Isso está coerente com a vasta infraestrutura de ciência e tecnologia da cidade de São Carlos, que, como já exposto na Seção 3.2, abriga centros de pesquisa e duas importantes universidades orientadas para atividades tecnológicas.

Pode-se destacar que as empresas estudadas, para desenvolverem novos produtos, mantêm em maior proporção interações verticais, como caracterizado por Lundvall (1988), com fornecedores de componentes, produtores de equipamentos médicos e clientes. Já as relações horizontais entre os produtores locais são praticamente inexistentes. E como não há, além de uma aglomeração geográfica, relacionamento sistêmico entre as empresas produtoras de equipamentos médicohospitalares, as universidades e o governo local o que caracterizaria um cluster, segundo Altenburg \& Meyer-Stamer (1999), apud Amato \& Garcia (2003) -, não se pode tratar o conjunto das empresas como um "arranjo produtivo local".

Concluindo este artigo, propomos como hipótese um padrão predominante de comportamento tecnológico para as empresas investigadas. Qualquer que tenha sido a fonte primária da tecnologia (matriz) - pesquisas nas universidades, institutos ou em outras empresas que geram spinoffs, engenharia reversa, licenciamento - que dá origem à empresa de base tecnológica e ao(s) produto(s) inicialmente comercializado(s) por ela, seu desenvolvimento subseqüente e sua manutenção no(s) nicho(s) de mercado penetrado -, possivelmente pela produção em pequena escala (e sob encomenda) de equipamentos ou instrumentos médicos e odontológicos (provavelmente customizados), que antes, no Brasil, eram somente importados, dependem da realização de um complexo conjunto de atividades tecnológicas.

Para desenvolvê-las, com um número muito pequeno de funcionários (técnicos e engenheiros), os proprietários ou diretores precisam manter contatos e relações freqüentes com clientes, principalmente, e com fornecedores, universidades e institutos de pesquisa. Os contatos com os clientes são imprescindíveis à especificação 
e ao processo posterior de avaliação e aperfeiçoamento dos produtos. Com os fornecedores, a relação garante acesso à tecnologia sobre insumos essenciais aos produtos e sobre etapas e operações dos processos de produção. Com áreas e laboratórios específicos das universidades e dos institutos de pesquisa, a relação possibilita resolver problemas identificados nos produtos e nos processos e dar continuidade ao acesso às tecnologias em desenvolvimento.

Nas pequenas empresas, com quadros reduzidos de funcionários para realizar essas e demais atividades, o tino empreendedor de proprietários e diretores e a existência de uma estrutura institucional, como a descrita na Seção 3, parecem constituir condições fundamentais ao comportamento tecnológico identificado. Portanto, algumas das condições para desenvolvimento de um arranjo produtivo local estão dadas.

Tal padrão comportamental encaixa-se na descrição do primeiro dos três estágios propostos por Utterback \& Abernathy (1975), ao analisarem a evolução do comportamento inovativo de pequenas empresas. É interessante notar que o comportamento no primeiro estágio de desenvolvimento é proposto como típico de pequenas empresas de base tecnológica (com altas taxas de crescimento) de setores dinâmicos nos países desenvolvidos. O comportamento descrito como de empresas no primeiro estágio poderia, então, também ser característico de empresas do setor de equipamentos médico-hospitalares e, possivelmente, de pequenas empresas de outros setores cujas características no cenário nacional são de indústrias nascentes e relativamente dinâmicas.

O teste da hipótese proposta e a continuidade dessa linha de argumentação dependem da obtenção de informações semelhantes às aqui trabalhadas, de natureza quantitativa, em um número maior de empresas do setor médico-hospitalar - o que constitui nossa próxima etapa de pesquisa - e em outros setores relativamente dinâmicos no panorama nacional. Além disso, explicação mais detalhada das diversas estratégias e dos comportamentos tecnológicos das empresas, que envolvem questões que aqui foram apenas resvaladas, dependem de estudos que contemplem informações de natureza qualitativa sobre as empresas, os segmentos de mercado, as tecnologias envolvidas, os comportamentos dos diretores e as relações que mantêm com clientes, fornecedores, universidades, institutos de pesquisa e agências de coordenação e fomento ao desenvolvimento tecnológico.

\section{Referências Bibliográficas}

ALTENBURG, T.; MEYER-STAMER, J. How to promote clusters: experiences from Latin America. World Development, v. 27, n. 9, 1999.

AMATO NETO, J.; GARCIA, R. Sistemas locais de produção: em busca de um referencial teórico. In: ENEGEP, 23., out. 2003, Ouro Preto. Anais... Ouro Preto, 1995.

CLASSIFICAÇÃO NACIONAL DAS ATIVIDADES ECONÔMICAS, 1997.

FERNANDES, A. C.; CÔRTES, M. R. Caracterização do Perfil da Pequena Empresa de Base Tecnológica no Estado de São Paulo: uma análise preliminar. São Carlos: UFSCar, 1998. Mimeo. (Relatório de Pesquisa.)
FERNANDES, A. C.; CÔRTES, M. R.; PINHO, M. S.; CARVALHO, R. Q. Potencialidades e limites para o desenvolvimento de empresas de base tecnológica no Brasil: contribuições para uma política setorial. 2000. Relatório $\mathrm{n}^{\mathrm{o}} 1$ referente ao Projeto FAPESP no 1998/14127-0.

FURTADO, J. A indústria de equipamentos médicohospitalares: elementos para uma caracterização da sua dimensão internacional. 1999. (no prelo.)

FURTADO, A. T.; SOUZA, J. H. Setor de equipamentos médicos - evolução do setor de insumos e equipamentos médico-hospitalares, laboratoriais e odontológico brasileiro: a década de 90 . Campinas, jun. 2000. 
GUIA DE FORNECEDORES HOSPITALARES. Hospitalar 2000, o maior evento da América Latina. São Paulo: Editora Guia de Fornecedores, maio 2000.

LAMBE, C. J.; SPEKMAN, R. E. Alliances, External Technology Acquisition, and Discontinuous Technological Change. Journal Prod. Innovation Management, v. 14, p. 102-116, 1997.

LANCOT, A.; SWAN, K. S. Technology Acquisition Strategy in an Internationally Competitive Environment. Journal of International Management, v. 6, p. 187-215, 2000. (2000 Elsevier Science Inc.)

PAVITT, K. Sectorial patterns of technical change: towards a taxonomy and a theory. Research Policy, v. 13, p. 343-373, 1984.

QUADOS DE CARVALHO, R.; FURTADO, A.; BERNARDES, R.; FRANCO, E. Technological innovation in Brazilian industry: an assessment based on the São Paulo innovation survey. In: International Conference on Technology Policy and Innovation, 3., Austin, 30 Aug.-2 Sep. 1999.

SEADE - FUNDAÇÃO SISTEMA ESTADUAL DE ANÁLISE DE DADOS. Questionário Indústria, Bloco 2 - PAEP (Pesquisa da Atividade Econômica Paulista), 1998.

SOBEET - SOCIEDADE BRASILEIRA DE ESTUDOS DE EMPRESAS TRANSACIONAIS E DA GLOBALIZAÇÃO ECONÔMICA. Comportamento Tecnológico das Empresas Transacionais em Operação no Brasil. Conjuntura Econômica, mar. 2000.
SUZIGAN, W. A indústria brasileira após uma década de estagnação: questões para política industrial. Economia e Sociedade, Campinas: IE-Unicamp, n. 1, p. 89-109, 1992.

TELLES, L. O. Cluster e a indústria ligada à área da saúde em Ribeirão Preto. 2002. Dissertação (Mestrado) - USP, Ribeirão Preto.

TORKOMIAN, A. L. Gestão da tecnologia na pesquisa acadêmica: o caso de São Carlos. 1997. Tese (Doutorado) - USP, Faculdade de Economia, Administração e Contabilidade.

UTTERBACK, J. M.; ABERNATHY, W. J. A dynamic model of process and product innovation. Omega, v. 3, 1975.

WILBON, A. D. An empirical investigation of technology strategy in computer software initial public offering firms. Journal of Eng. Technology Management, v. 16, p. 147-169, 1999.

ZAHRA, S. A. Technology strategy and financial performance: examining the moderating role of the firm's competitive environment. Journal of Business Venturing, v. 11, p. 189-219, 1996.

ZAHRA, S. A. Technology strategy and new venture performance: a study of corporate sponsored and independent biotechnology ventures. Journal of Business Venturing, v. 11, p. 289-321, $1996 \mathrm{~b}$.

ZAHRA, S. A.; BOGNER, W. C. Technology strategy and software new ventures' performance: exploring the moderating effect of the competitive environment. Journal of Business Venturing, v. 15, p. 135-173, 1999.

\title{
TECHNOLOGICAL EFFORTS AND INNOVATION PERFORMANCE OF MEDICAL EQUIPMENT FIRMS LOCATED IN SÃO CARLOS - STATE OF SÃO PAULO
}

\begin{abstract}
This paper joins information on technological efforts and innovation performance of 12 medical equipment producers located in São Carlos, State of São Paulo. It was gathered through interviews with owners or managers in the firms, who are asked to answer a structured questionnaire. Our analysis of this information shows that those firms have been done considerable efforts directed toward both internal $R \& D$ - in higher proportion - and technology acquisition from external sources. The majority of the firms have not invested on technology acquisition from external sources more than one third of their investment on internal $R \& D$. Considering innovation performance, $R \& D$ activities in those firms are mainly oriented to incremental product innovations.
\end{abstract}

Key words: technological efforts, innovation performance, São Carlos City, medical equipment firms. 


\section{Apêndice 1 \\ Indicadores de esforço tecnológico e desempenho inovador Esforço Tecnológico}

- Existência de departamento de P\&D estruturado ou semi-estruturado dentro da empresa.

- Gastos com atividades internas de P\&D em relação às vendas (ou faturamento) da empresa (média dos últimos três anos).

- Porcentagem das despesas com P\&D destinadas a inovações em produto.

- Porcentagem das despesas com P\&D destinadas a inovações em processo.

- Número de técnicos de nível médio no P\&D (média dos últimos três anos).

- Número de engenheiros (ou cientistas) no P\&D (média dos últimos três anos).

- Freqüência com que a empresa contrata outras empresas, universidades ou centros de pesquisa para desenvolver tecnologias de produto e processo.

- Freqüência com que a empresa faz uso de alianças estratégicas com consumidores, fornecedores, outras empresas nacionais e estrangeiras, universidades e centros de pesquisa para desenvolver produtos e processos tecnologicamente novos ou melhorados.

- Freqüência com que a empresa compra ou licencia tecnologias de produto e processo desenvolvidas por outras empresas para incorporar em seus próprios produtos e processos.

- Capital fixo investido em aquisição de tecnologias de produto e processo externamente à empresa em relação às vendas (ou faturamento) da empresa nos últimos três anos.

- Gastos com patentes, copyright e trademarks em relação às vendas (ou faturamento) da empresa (média dos últimos três anos).

\section{Desempenho Inovador}

- Número aproximado de inovações de produtos de natureza incremental e de natureza significativa que a empresa tem introduzido no mercado nos últimos três anos.

- Número aproximado de inovações em processos de natureza incremental e de natureza significativa que a empresa tem desenvolvido e utilizado nos últimos três anos.

- Parcela do faturamento derivada de novos produtos ou produtos melhorados desenvolvidos internamente à empresa (não-licenciados) e introduzidos no mercado nos últimos três anos. 


\section{Apêndice 2}

\section{Questionário parcial}

\section{Composição da empresa em termos de funcionários}

\begin{tabular}{|l|l|}
\hline Total de funcionários & \\
\hline Total de técnicos de nível médio & \\
\hline $\begin{array}{l}\text { Total de funcionários com algum tipo de formação } \\
\text { acadêmica (graduação e pós-graduação) }\end{array}$ & \\
\hline Total de funcionários na produção & \\
\hline Total de técnicos de nível médio na produção & \\
\hline Total de funcionários na administração & \\
\hline
\end{tabular}

* Técnico de nível médio: pessoal formado em curso técnico.

\section{Principais linhas de produtos e participação no faturamento anual da empresa}

\begin{tabular}{|l|l|}
\hline Linha de produto & $\%$ do faturamento anual \\
\hline & \\
\hline & \\
\hline
\end{tabular}

Registro no Ministério da Saúde?

\section{Identificação de estratégias tecnológicas}

Circule o número que melhor descreva a situação de sua empresa nos últimos três anos usando a escala abaixo (questões 1 a 9).

$\begin{array}{lccccc}1 & 2 & 3 & 4 & 5 & \text { NA } \\ \text { ito baixo } & \text { Baixo } & \text { Médio } & \text { Alto } & \text { Muito alto } & \text { Não se aplica }\end{array}$

1. A frequiência na qual sua empresa contrata outras empresas, universidades ou centros de pesquisa para desenvolver tecnologias de produto e processo.

$\begin{array}{lllllll}\text { Produto } & 1 & 2 & 3 & 4 & 5 & \text { NA } \\ \text { Processo } & 1 & 2 & 3 & 4 & 5 & \text { NA }\end{array}$

A frequiência na qual sua empresa faz uso de...

2. ...alianças com consumidores para desenvolver produtos e processos tecnologicamente novos ou melhorados.

$\begin{array}{lllllll}\text { Produto } & 1 & 2 & 3 & 4 & 5 & \text { NA } \\ \text { Processo } & 1 & 2 & 3 & 4 & 5 & \text { NA }\end{array}$

3. ...alianças com fornecedores para desenvolver produtos e processos tecnologicamente novos ou melhorados.

$\begin{array}{lllllll}\text { Produto } & 1 & 2 & 3 & 4 & 5 & \text { NA } \\ \text { Processo } & 1 & 2 & 3 & 4 & 5 & \text { NA }\end{array}$


4. ...alianças com empresas estrangeiras para desenvolver produtos e processos tecnologicamente novos ou melhorados.

$\begin{array}{lllllll}\text { Produto } & 1 & 2 & 3 & 4 & 5 & \text { NA } \\ \text { Processo } & 1 & 2 & 3 & 4 & 5 & \text { NA }\end{array}$

5. ...alianças com outras empresas nacionais para desenvolver produtos e processos tecnologicamente novos ou melhorados.

$\begin{array}{lllllll}\text { Produto } & 1 & 2 & 3 & 4 & 5 & \text { NA } \\ \text { Processo } & 1 & 2 & 3 & 4 & 5 & \text { NA }\end{array}$

6. ...alianças com universidades ou centros de pesquisa para desenvolver produtos e processos tecnologicamente novos ou melhorados.

$\begin{array}{lllllll}\text { Produto } & 1 & 2 & 3 & 4 & 5 & \text { NA } \\ \text { Processo } & 1 & 2 & 3 & 4 & 5 & \text { NA }\end{array}$

7. Freqüência na qual sua empresa compra ou licencia tecnologias de produto e processo desenvolvidas por outras empresas para incorporar em seus próprios produtos e processos.

$\begin{array}{lllllll}\text { Tecnologia de produto } & 1 & 2 & 3 & 4 & 5 & \text { NA } \\ \text { Tecnologia de processo } & 1 & 2 & 3 & 4 & 5 & \text { NA }\end{array}$

8. Freqüência na qual a compra e o licenciamento de tecnologias de produto e processo são seguidos de atividades de adaptação e melhorias do que foi efetivamente comprado.

$\begin{array}{llllllll}\text { Tecnologia de produto } & 1 & 2 & 3 & 4 & 5 & \text { NA }\end{array}$

$\begin{array}{lllllll}\text { Tecnologia de processo } & 1 & 2 & 3 & 4 & 5 & \text { NA }\end{array}$

9. A empresa possui departamento de Pesquisa e Desenvolvimento (P\&D)?

( ) Estruturado （ ) Semi-estruturado （） Não possui P\&D

Se possuir P\&D estruturado ou semi-estruturado, indicar (questões 10 a 22):

10. Gastos com atividades internas de $\mathrm{P} \& \mathrm{D}$ em relação às vendas (ou faturamento) da empresa (média dos últimos três anos): $\%$

11. Porcentagem das despesas em $P \& D$ destinadas a inovações em produto: $\%$

12. Porcentagem das despesas em P\&D destinadas a inovações em processo: $\%$

13. Sobre o número de funcionários:

\begin{tabular}{|l|l|}
\hline Total de funcionários alocados à P\&D & \\
\hline Total de engenheiros e cientistas alocados à P\&D & \\
\hline Total de técnicos de nível médio alocados à P\&D & \\
\hline
\end{tabular}

14. Capital fixo investido na aquisição de tecnologias de produto e processo a partir de fontes externas à empresa. (Está incluído aqui o capital fixo investido em alianças estratégicas e contratações de universidades, centros de pesquisa, outras empresas ou instituições governamentais para desenvolvimento de tecnologias de produto ou processo e para compra ou licenciamento de novas tecnologias de produto e processo.): $\%$

15. Enumere de 1 a 4 aquelas atividades que representam as maiores (1) e as menores (4) parcelas dos investimentos feitos na aquisição externa de tecnologias de produto e processo: 
( ) Capital fixo investido em alianças estratégicas para desenvolvimento de novas tecnologias de produto e processo.

( ) Capital fixo investido em contratações de centros de pesquisa, universidades, empresas e instituições governamentais para desenvolvimento de novas tecnologias de produto e processo.

( ) Capital fixo investido na compra de tecnologias de produto e processo desenvolvidas por outras empresas.

( ) Capital fixo investido em licenciamento de tecnologias de produto e processo disponíveis no mercado.

16. Gastos com patentes em relação às vendas (ou faturamento) da empresa (média dos últimos três anos): $\%$.

17. Número aproximado de inovações de produtos de natureza significativa que a sua empresa tem introduzido no mercado nos últimos três anos:

18. Número aproximado de inovações de produtos de natureza incremental que a sua empresa tem introduzido no mercado nos últimos três anos:

19. Número aproximado de patentes de produto solicitadas ou obtidas por sua empresa nos últimos cinco anos:

20. Parcela do faturamento derivada de novos produtos ou de produtos melhorados desenvolvidos internamente à empresa (não-licenciados) e introduzidos no mercado nos últimos três anos: $\%$.

21. Número aproximado de inovações em processos de natureza significativa que a empresa tem introduzido nos últimos três anos:

22. Número aproximado de inovações em processos de natureza incremental (melhorias) que a empresa tem introduzido nos últimos três anos: 